\title{
Modeling local terrain attributes in landscape-scale site-specific data using spatially lagged independent variable via cross regression
}

\author{
by Griffin, T. and Lowenberg-Deboer, J.
}

Copyright, publisher and additional information: .This is the authors' accepted manuscript. The published version is available via Springer.

Please refer to any applicable terms of use of the publisher.

DOI: https://doi.org/10.1007/s11119-019-09702-5 


\section{Precision Agriculture}

\section{Modeling local terrain attributes in landscape-scale site-specific data using spatially lagged independent variables via cross regression

\author{
--Manuscript Draft--
}

\begin{tabular}{|l|l|}
\hline Manuscript Number: & PRAG-D-18-00135R3 \\
\hline Full Title: & $\begin{array}{l}\text { Modeling local terrain attributes in landscape-scale site-specific data using spatially } \\
\text { lagged independent variables via cross regression }\end{array}$ \\
\hline Article Type: & Manuscript \\
\hline Keywords: & $\begin{array}{l}\text { cross regression; elevation; landscape position; lagged independent variable; } \\
\text { spatial; topography }\end{array}$ \\
\hline Corresponding Author: & $\begin{array}{l}\text { Terry Griffin, PhD } \\
\text { Kansas State University } \\
\text { UNITED STATES }\end{array}$ \\
\hline Corresponding Author's Institution: & Kansas State University \\
\hline First Author: & Terry Griffin, PhD \\
\hline Order of Authors: & Terry Griffin, PhD \\
\hline & James Lowenberg-DeBoer \\
\hline Funding Information: & \\
\hline
\end{tabular}


 \\ Modeling local terrain attributes in landscape-scale site-specific data using spatially lagged independent variable via cross regression
}

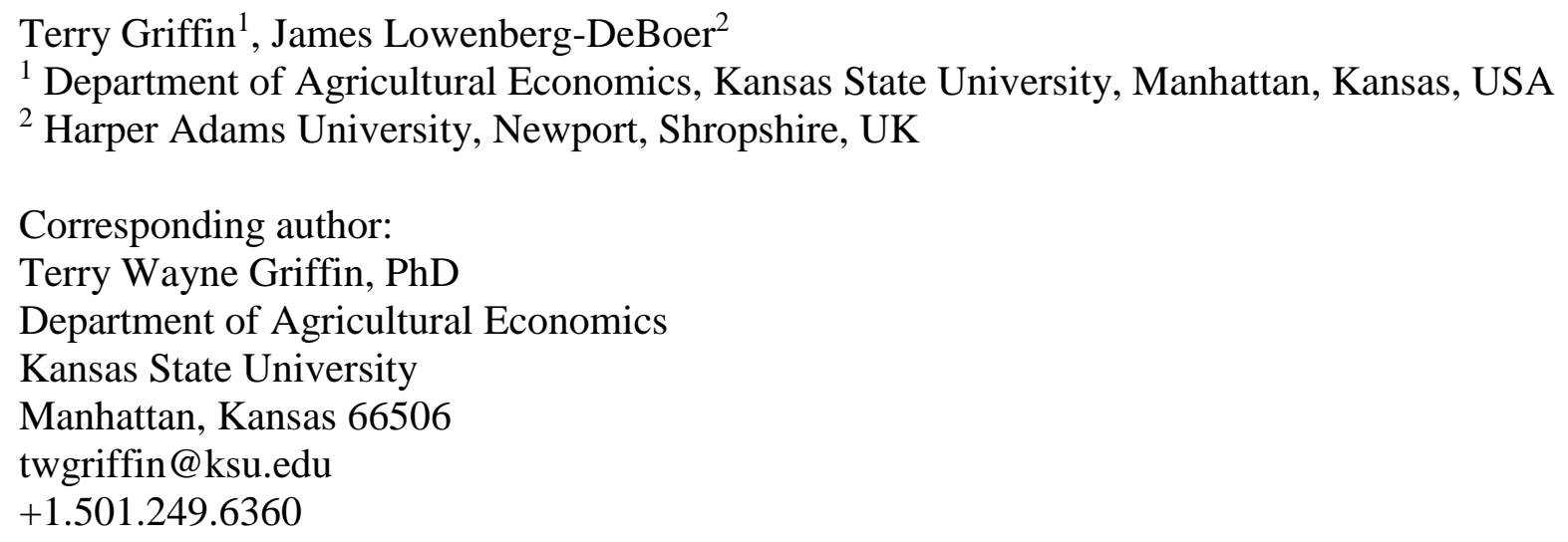

\begin{abstract}
Analysis methods for landscape-scale site-specific agricultural datasets have been adapted from a wide range of quantitative disciplines. Due to spatial effects expected at landscape scales with respect to yield affecting factors, inference from aspatial analyses may lead to inefficient statistical inference. When spatial correlation exists within a random variable e.g. explanatory variables such as elevation or soil characteristics, spatial statistical methods can provide unbiased and efficient estimates on which to base economic analyses and farm management decisions. Simple continuous terrain variables derived from spatially lagged independent variable transformation of relative terrain position allowed models to be estimated using familiar linear aspatial models without introducing the problems associated with interpolated data in inferential spatial statistics. Using site-specific data from three example fields, cross regressive elevation variables complemented topographic attributes, rather than replacing them in a range of statistical models. Results indicated that cross regressive elevation variables, especially relative elevation, reduced estimation problems due to correlation among independent variables and bias arising from spatially interpolated data in statistical analysis.
\end{abstract}

Keywords: cross regression, elevation, landscape position, lagged independent variable 


\section{Introduction}

The advent of global navigation satellite systems (GNSS) empowered farmers to test input choices before implementing farm management decisions across larger areas. Farmers are making decisions based on analysis of yield monitor data (Griffin et al. 2008). Data from yield monitors motivated the resurgence of on-farm experimentation because farmers could measure yield responses without interfering with harvest-time field operations (Griffin et al. 2014). Recent studies estimate $39 \%$ and $68 \%$ of midwestern US farms have georeferenced yield data and automated guidance, respectively (Griffin \& Yeager 2019; Miller et al 2019). Farmers with GNSS-enabled yield monitors are likely to conduct landscape-scale on-farm experiments (Griffin 2010). Technology-endowed farms are candidates for utilizing the analysis tools presented in this study. Farms with either GNSS-enabled yield monitors or automated guidance are likely to have access to elevation data sufficient to make use of these analyses. In addition, farms without high accuracy GNSS elevation data may have light detection and ranging (LiDAR) data available at near zero cost (Thomas et al., 2017). The overall objective of this study was to determine if microscale landscape position variables based on cross regression can add to the explanatory power of regression-based analysis of crop sensor data. While farmers are typically not fixated on statistical testing in the same way that researchers are, they are concerned about the reliability of the results. Statistical inference analysis is the most likely basis for reliability indicators in farm decision support tools. These tools are more likely utilized within automated cloudcomputing routines for farm-level data analysis rather than farmers interacting via desktop software. The advent of Big Data has encouraged researchers and agricultural entrepreneurs to develop automated decision-making tools (Coble et al., 2018).

Supporting factors of production are needed for inference from analyzing yield monitor data. Explanatory variables include treatment information from deliberate interventions recombined with environmental soil characteristics such as elevation and terrain attributes. Landscape position is known to influence crop productivity, variability and yield response to input application. Simple searches within Precision Agriculture journal returned 206, 135 and 193 articles for elevation, terrain and topography, respectively.

Topographic modeling techniques applied to statistical models include hydrologic models, indices of variables, digital elevation models (DEM), elevation as simple covariates and derivatives of elevation surfaces such as slope, aspect and curvature. Even though elevation and 
derivatives of elevation may have no direct interpretation with respect to crop yield, elevationderived covariates typically explain substantial portions of the noise component of the model, earning its place as one of most common topography variable regimes in the literature. These noise-explaining variables are likely to proxy for environmental properties that do influence crop growth and yield including total wetness and other factors that affect water availability. Additional advantages of elevation data include continual collection of data with nearly every equipment pass during field operations and regionally accessible publicly available LiDAR. A secondary benefit of GNSS-enabled automated guidance is accurate, high resolution and lowcost elevation data collected during most field operations.

Omitting variables important to statistical model specification leads to several problems influencing inference. Including variables measured incorrectly leads to errors in variables problems. These statistical failures with respect to topography, omitted variable or errors in variables, may be prevented with appropriate spatial techniques. A method to create a relative terrain position, i.e. relative elevation, via cross-regressive techniques requiring no spatial interpolation is proposed. The proposed cross-regressive models are compared to the more common spatial error process models that tend to be favored by many researchers seeking inferential statistics for yield response in field-scale experimentation. Some researchers, especially those with roots in geography or economics, favor the spatial error or spatial lag regression models for statistical inference (e.g. Anselin et al., 2004; Florax et al, 2002; Griffin et al., 2008; Hurley et al., 2005). Others, especially those coming from crop science or soil science, favor nearest neighbor analyses originally suggested by Papadakis (1937). Lambert et al. (2004) compared the most common spatial error process models, showed that they have a common theoretical base and provided empirical examples in which all the spatial error process models resulted in similar conclusions, which were quite different from the results of aspatial analysis.

The overall objective of this study was to determine if microscale landscape position variables based on cross regression can add to the explanatory power of regression-based analysis of crop sensor data. The spatial error process model tends to be the standard model selected by the majority of researchers evaluating field-scale experiments. The simpler cross regressive model may be a viable alternative especially when topographic variables are included on the righthand side of the regression equation. The specific objectives were to determine in example data sets: 1) if cross regression elevation variables create multicollinearity problems in estimation, and 2) if 
they avoid the statistical problems of using interpolated values as independent variables. Using spatial inferential statistics (i.e. spatial econometrics) that model local spatial autocorrelation, relative elevation, slope and overall micro-scale landscape position are used to model yields with a limited number of continuous covariates. Hypotheses include 1) model specifications including cross regression relative elevation variables facilitate estimation of treatment differences or optimal input rates and 2) model specification with the proposed cross regressive elevation variable does not affect the multicollinearity condition number. Multicollinearity is the intercorrelation among explanatory variables in a regression model (Greene 2012). Multicollinearity is measured by condition number $(\mathrm{CN})$ of the matrix of explanatory variables. Condition number is the ratio of largest and smallest eigenvalues of the matrix (Greene, 2012).

Spatial regression techniques modeled relative elevation, slope, overall micro-scale landscape position and local spatial autocorrection with a limited number of covariates. Implications of differing topography variables for spatial data analysis of field-scale on-farm comparisons were demonstrated. Effectiveness of various alternative specifications were assessed. Field scale experiments were managed by farmers in collaboration with the authors (Griffin et al. 2008). Research questions, treatments tested and experimental designs were chosen by farmers with guidance from the authors. This study builds upon Griffin et al. (2008) by updating the methods and applying tools to a wide range of data in development of automated tools for spatial data analysis and decision making.

\section{Background}

Agricultural productivity is influenced by terrain position. Elevation and other topographic information have been used in precision agriculture studies for three broad categories, 1) identification of management zones, 2) empirical crop modeling and 3) soil mapping (Bishop and McBratney 2002). Their third category is evident with USDA-NRCS soil mapping units being defined by slope class categories. Category 1 and category 2 are of interest to farmers now that elevation data are easily collected at relatively low cost. Although the highest accuracy GNSS are preferred to produce topographic maps (Clark and Lee, 1998), recent agricultural technology innovations for data gathering (e.g. combine yield monitors and other site-specific sensors) and navigation (e.g. lightbars and automated guidance) may provide sufficiently accurate elevation measurements for use as covariates in statistical models (Garrido et al., 2019). 
Elevation data is important in estimation of treatment effects in datasets acquired from fields with micro-scale topography differences. In cases such as precision leveled fields in flood irrigated crops such as rice, elevation would not be considered an important covariate. However, for broad area wheat, soybean and corn production, local terrain attributes may play a substantial role (Griffin et al. 2008).

Although elevation has been successfully used as a covariate in field-scale precision agriculture datasets, the elevation variable alone cannot adequately model the relative terrain position for an observation. Even within the same field, an elevation measurement of say $200 \mathrm{~m}$ may be 1) on a hilltop, 2) valley bottom and 3) hill slope. Advanced elevation modeling techniques interpolate elevation into a so-called digital elevation model (DEM) surface from which slope and other elevation derivatives can be calculated, e.g. plan curvature, profile curvature and aspect. Although spatial interpolation has its place and elevation derivatives have been useful for many soils and crop modeling procedures, they may not be as useful in statistical modeling of on-farm research for two reasons. The first reason is that the elevation measurements must be interpolated on to a surface thus introducing a systematic error into the data (Anselin 2001). Unlike random errors, systematic errors affect the average of the explanatory variable and biases the estimated coefficient. Second, estimation of regression models suffers from too many continuous covariates especially when several variables are linear transformations, i.e. linearly or non-linearly dependent, of one another resulting in multicollinearity. A smaller number of variables that model relative terrain position without introducing systematic errors of spatial interpolation would be useful to spatial analysis of landscape-scale precision agriculture datasets.

If slope or other variables created from an interpolation process are conceptually important to the statistical model, an omitted variable problem results from exclusion potentially leading to biased estimated coefficients. Conversely, if an interpolated surface from sparse data layers (e.g. soil fertility measurements), are used as explanatory variables, errors in variables may result. If these important spatially autocorrelated explanatory variables are not available in precision agriculture datasets, omitted variables problems result. For instance, many farmers collect supporting information at scales beyond the spatial range, e.g. phosphorus and potassium samples are commonly taken from 1-ha grid sizes resulting in observations being no closer than $100 \mathrm{~m}$. These distances often exceed the spatial range resulting in no spatial autocorrelation 
detected in the data; thus, typical soil fertility measurements are not conducive for spatial analysis. However, it is feasible for some factors to be measured at relatively higher resolutions.

Elevation is an example of a feasible relatively dense measurement. Geolocated technology innovations for data gathering (e.g. combine yield monitors and other sensors) and navigation (e.g. automated guidance) provide elevation measurements. Previous studies have shown elevation effects on crop response (Jiang and Thelan, 2004; Kaspar et al., 2004; Kravchenko et al., 2000) and others included elevation and other topographic measurements as explanatory variables for regression models (Anselin et al., 2004; Miao et al., 2006; Hartsock et al., 2005). Long and McCallum (2015) analyzed yield monitor data and LiDAR for wheat research. Topographical data is useful to delineate zones with crop sensitivity to environmental factors (Kravchenko et al., 2000). Elevation data and derivations including slope, aspect and curvature have been used as covariates; however, slope and other elevation surface derivatives rely upon spatial interpolation. To calculate elevation derivatives, elevation data must be interpolated on to a smooth surface, the so-called digital elevation model (DEM). From the elevation surface, slope calculations are based on simple calculus; however, the process of interpolating a finite set of elevation measurements on to a smooth surface introduces a random variable with a systematic error (Anselin, 2001).

One potential method to avoid errors in variables and omitted variable problems is spatiallylagged independent variable models, i.e. cross regression (Arbia 2014). Cross-regressive models utilize spatially-lagged independent variable(s) including spatially-weighted exogenous variables on the right-hand side that can be estimated as ordinary least squares (OLS) (Anselin 2002; Arbia 2014; Florax and Folmer 1992). Cross-regressive models are an extension of familiar aspatial linear models (Eq. 1),

$$
\boldsymbol{y}=\boldsymbol{X \beta}+\boldsymbol{\mu}
$$

where $\mathbf{y}$ is an $n \times \mathbf{x} 1$ vector of observations on the dependent variable, $\mathbf{X}$ is an $n$ by $k$ matrix of explanatory variables, $\boldsymbol{\beta}$ is an $k$ by 1 vector of regression coefficients and $\boldsymbol{\mu}$ an independently and identically distributed error term. Arbia (2014) presented the general form of the linear spatial regression model and five special cases (p 51-52, note that Arbia used the phrase "remarkable cases"). 


$$
\begin{array}{ll}
\boldsymbol{y}=\lambda \boldsymbol{W} \boldsymbol{y}+\boldsymbol{X} \boldsymbol{\beta}_{(1)}+\boldsymbol{W} \boldsymbol{X} \boldsymbol{\beta}_{(2)}+\boldsymbol{\mu} & |\lambda|<1 \\
\boldsymbol{\mu}=\rho \boldsymbol{W} \boldsymbol{\mu}+\boldsymbol{\varepsilon} & |\rho|<1
\end{array}
$$

where $\mathbf{X}$ is a matrix of non-stochastic regressors, $\mathbf{W}$ is an exogenously defined row-standardized $n$ by $n$ spatial weights matrix, $\boldsymbol{\varepsilon} \mid \boldsymbol{X} \approx$ i.i.d.N $\left(0, \sigma_{\varepsilon}^{2}{ }_{n} \mathbf{I}_{n}\right)$ and $\boldsymbol{\beta}_{1}, \boldsymbol{\beta}_{2}, \lambda$ and $\rho$ parameters to be estimated (Arbia 2014 page 51). Rewriting Eq. 2 as

$\boldsymbol{y}=\lambda \boldsymbol{W} \boldsymbol{y}+\boldsymbol{Z X \boldsymbol { \beta }}+\boldsymbol{\mu} \quad|\lambda|<1$

such that $\boldsymbol{Z}=[\boldsymbol{X}, \boldsymbol{W} \boldsymbol{X}]$ and $\boldsymbol{\beta}=\left[\boldsymbol{\beta}_{(1)}, \boldsymbol{\beta}_{(2)}\right]$ (Arbia 2014 page 52). Five special cases are derived from the spatial autoregressive model with additional autoregressive error structure (SARAR) (Anselin, 1988; Arbia 2014; Kelejian and Prucha 1998). Arbia (2014, page 52) presented these five special cases as

(i) $\quad \boldsymbol{\beta}=0$ and either $\lambda$ or $\rho=0$, the pure spatial autoregressive model

(ii) $\lambda=\rho=0$, the lagged independent variable model

(iii) $\lambda=0, \rho \neq 0$, the spatial lag model (SLM)

(iv) $\lambda \neq 0, \rho=0$, the spatial error model (SEM)

(v) $\lambda \neq 0, \rho \neq 0$, the complete spatial model (SARAR)

This study applied the second and fourth special cases to topography attributes. The second special case includes spatially-lagged independent variables and is sometimes referred to as the cross-regressive model with one or more cross-regressive variables WX. It is assumed $\boldsymbol{Z}=$ $[\boldsymbol{X}, \boldsymbol{W} \boldsymbol{X}]$ is full rank such that $\mathbf{Z}$ may contain a spatial lag of some or all the independent variables. Cross-regressive models are estimated as OLS and intended to explicitly account for local spatial externalities and given as Eq. 5

$$
y=X \beta+W Z \gamma+\mu
$$


where $\mathbf{Z}$ is a $k$ by $n$ matrix of $k$ explanatory variables that can be the same as $\mathbf{X}$ except without the intercept term, $\gamma$ is a $k$ by 1 vector of regression coefficients on the cross-regressive term $\mathbf{W Z}$ and remaining terms have been previously defined (Arbia 2014).

Cross-regression explicitly models local spillovers. In applications of spatial statistical techniques applied to precision agriculture cases, spatial spillover effects have almost exclusively been modeled as global processes, where 'global' refers to each location in the field being linked to any other location in the field. Global linkage processes are inherent to the frequently used spatial autoregressive models. Local spatial spillovers exist with only immediately adjacent observations. As an example, measurement errors are likely with precision agriculture sensors and these errors tend to "spill over" across spatial units. The errors for spatial unit $i$ are likely to be correlated to the errors in a neighboring unit $j$; spatial dependence may be caused by these spatial spillovers (Anselin, 1988). In on-farm experimentation, the local spillover effect may include treatment edge effects where treatments applied to neighboring spatial units impact yield response in adjacent spatial units. When true model specifications include $\mathbf{W Z}$ terms but estimated as OLS without lagged independent variables, the estimated coefficients remained unbiased and efficient. Cross-regressive variables have rarely been used in production agriculture. An exhaustive review of the literature revealed no other mention of cross-regressive or spatially lagged independent variables for production agriculture especially with respect to analysis of site-specific data.

Spatially-weighted exogenous variables can be included in linear aspatial and spatial process models such as spatial error models (SEM). The SEM (sometimes referred to as spatial autoregressive or SAR) explicitly models spatial autocorrelation in the error term, $\boldsymbol{\mu}$. Sitespecific data collected from landscape scale on-farm experiments are expected to have spatial effects such as dependence and autocorrelation. Given statistical failures, these data analyses likely benefit from spatial error process models due to omitted variable (e.g. subsoil characteristics, microclimate), rather than contagion within dependent variables. Omitting an important variable with its own spatial effects causes aspatial model residuals to be spatially autocorrelated. Diagnostics evaluating OLS residuals empirically test for spatial effects in residuals and dependent variable. These diagnostics provide quantitative insights into selection of the most appropriate spatial process model.

The fourth special case described by Arbia (2014) is the SEM and given as 


$$
\begin{aligned}
& \qquad \begin{array}{l}
\boldsymbol{y}=\boldsymbol{X} \boldsymbol{\beta}+\boldsymbol{\varepsilon}, \quad \boldsymbol{\varepsilon}=\lambda \boldsymbol{W} \boldsymbol{\varepsilon}+\boldsymbol{\mu} \\
\text { or in reduced form as } \\
\boldsymbol{y}=\boldsymbol{X} \boldsymbol{\beta}+(\boldsymbol{I}-\lambda \boldsymbol{W})^{-1} \boldsymbol{\mu}
\end{array}
\end{aligned}
$$

where $\varepsilon$ is an $n$ by 1 vector of residuals, $\lambda$ a spatial autoregressive parameter, $\boldsymbol{\mu}$ a well behaved, non-heteroskedastic uncorrelated error term (Anselin 1988) and others as previously defined. The $(\boldsymbol{I}-\lambda \boldsymbol{W})^{-1}$ term is the spatial multiplier. When the spatial autoregressive term, $\lambda$, is 0 , the spatial error model reverts to the familiar aspatial linear model (Eq. 1), $\boldsymbol{y}=\boldsymbol{X} \boldsymbol{\beta}+\boldsymbol{\mu}$. The spatial error process can be characterized by the global spillovers due to spatial multipliers. When the spatial error model is appropriate, OLS estimators remain unbiased but are inefficient.

Comparison of spatial statistical methods have been conducted by simulation and field experimentation. Dubin (2003) stated geostatistical methods, which could be estimated as restricted maximum likelihood (REML), outperformed spatial process models when the true form of spatial variability was unknown. Conversely, several studies analyzing site-specific data determined SEM was an appropriate model. Spatial process models have been shown to provide a framework to appropriately model spatial effects (Anselin et al., 2004; Hurley et al., 2005; Liu et al., 2015; Long and McCallum, 2015; Trevisan et al., 2019). Anselin et al (2004) were likely the first to apply SEM to precision agriculture. They evaluated field-scale nitrogen fertilizer trials in Argentina and found aspatial models were not sufficient to address spatial effects. Lambert et al. (2004) compared ordinary least squares and four spatial regression methods on the Los Rosas dataset originally reported by Anselin et al., (2004). Lambert's study reported that all four spatial regression methods provided similar estimates, although spatial processes and geostatistical techniques were able to model the treatment effects better than methods that did not explicitly account for spatial structure in the data. Liu et al (2015) compared spatial process models to evaluate nematicides in cotton production. They reported that SEM model results were more practical to build university Extension recommendations than other candidate models. Advantages of the spatial process model include being conducted in a single step, estimated with fewer observations and able to model spatial autocorrelation in the dependent variable, error term 
or explanatory variables. The geostatistical approach estimated as REML is estimated in three steps, requires more observations and only applies to the error process. One criticism of the spatial process model is the exogenously-defined spatial interaction structure. Recently, Selle et al. (2019) suggested established models such as Integrated Nested Laplace Approximation (INLA) with Stochastic Partial Differential Equation (SPDE) could improve analysis of field experiments. Each of these statistical models are readily available in popular open source software environments. Spatial effects violating assumptions of classical statistics may be modeled in more than one method; these effects may be included as predictors in the model or could be explicitly modeled if properly parameterized and can have similar predictive power. In any case, these studies indicated that explicitly modeling spatial variability exhibited advantages over analyzing data with aspatial models.

\section{Methods}

Spatial analyses were conducted on landscape-scale on-farm experiments to demonstrate usefulness of alternative topographic variables. Cross-regressive variables, WZ, were created for each dataset to evaluate localized terrain effects on yield response to deliberate treatments. The first step was to choose the spatial interaction structure for use in calculating the spatiallyweighted elevation term. In general, spatial weights matrices were constructed such that $w_{i i}=0$, $w_{i j}>0$ for observations considered neighbors, and $w_{i j}=0$ for non-neighbors where $w$ is an element of $\mathbf{W}$ and $i j$ denotes the matrix position. Spatial weights matrices for local terrain effects (hereafter referred to as $\mathbf{W}_{1}$ ) were selected such that only immediately neighboring observations were of interest therefore specifications such as first-order queen contiguity or minimum Euclidean distances were considered. In either case, Boolean matrices were constructed with zeros as non-neighbors and ones as neighbors before row-standardizing.

The $n$ by 1 vector of continuous elevation data, $\mathbf{E}$, was pre-multiplied by the $n$ by $n$ spatial weights matrix, $\mathbf{W}_{\mathbf{1}}$, producing the $n$ by 1 cross-regressive term $\boldsymbol{W}_{1} \boldsymbol{E}$. Cross-regressive terms measure spatially weighted average elevation of immediate neighbors as defined by spatial interaction structure, $\mathbf{W}_{\mathbf{1}}$. The spatially weighted average elevation variables, $\boldsymbol{W}_{\mathbf{1}} \boldsymbol{E}$, results in a smoothed elevation variable. Rather than including smoothed elevation, $\boldsymbol{W}_{\mathbf{1}} \boldsymbol{E}$, was used to create a relative elevation variable. The cross-regressive term, $\boldsymbol{W}_{1} E$, was subtracted from the elevation value of observation in question providing relative elevation, $R E=E-\boldsymbol{W}_{\mathbf{1}} E$. 
Relative elevation, RE, captures localized terrain position for use in statistical models. When relative elevation is negative, $R E<0$, observations are lower in elevation than average of immediate neighbors. When relative elevation is positive, $R E>0$, observations are higher than spatially-weighted average of its neighbors. Observations are at the same elevation as the average of neighbors when equal to zero, $R E=0$. When $R E=0$, the observation could be on a flat plain or hillside such that average of the neighbors equates to elevation of observation. This is a known limitation of relative elevation variables compared to slope variables distinguishing observations on hillsides. However, observations with slope equal to zero are unable to be discerned between hilltop and valley. Relative elevation indicates direction of relative position and magnitude of differences. Since terrain slopes are generally calculated from interpolated elevation surfaces, the magnitude of $R E$ partially substitutes for slope allowing models to be estimated without systematic error associated with spatially interpolated values.

Aspatial, cross-regressive, and SEM models were estimated to analyze field-scale site-specific data. Yield monitor data were cleaned to remove erroneously measured observations and to relocate points to correct locations per procedures suggested by Griffin et al., (2007) and Sudduth et al. (2012). Aspatial and cross-regression analyses were estimated as OLS. Spatial error process models were estimated as general moments (GM) for all model specifications. General moments estimators were chosen due to large sample sizes of field experiments and no assurance of normal error distribution (Kelejian and Prucha 1999; Kelejian and Prucha 2010; Bell and Bockstael 2000; LeSage and Pace, 2009).

An inverse distance spatial weights matrix $w_{i j}=\frac{1}{d_{i j}}$ hereafter referred to as $\boldsymbol{W}_{2}$, was chosen to define the spatial interaction structure for SEM models. Each element of $\mathbf{W}_{2}$, $w_{i j}$, were calculated as the inverse of the distance, $d$, from i to $\mathrm{j}, w_{i j}=1 / d_{i j}$. Assigning weights based on inverse of proximity was chosen for the SEM model so that neighbors further away did not influence error process as much as nearby neighbors. Model specifications were evaluated by Akaike Information Criterion (AIC) (Anselin 1988; Greene 2012). The AIC degrades as model size increases, i.e. penalties placed on increased numbers of explanatory variables. The models were estimated as GM and sigma squared $\left(\hat{\sigma}_{k}^{2}\right)$ reported. The measure of fit was calculated as $A I C=N\left(\ln 2 \pi \hat{\sigma}_{k}^{2}+1\right)+k$, where $N$ was number of observations and $k$ number of variables. 


\section{Results}

Three example fields were evaluated and presented here. The first field presented, Field A, had no deliberate intervention. Field $\mathrm{B}$ and Field $\mathrm{C}$ included farmer-managed field-scale deliberate intervention research for categorical and continuous variables, respectively. The rate trial was a soybean seeding rate study (Field B hereafter referred to as SOYSEED). The categorical trial included pesticide treatments applied to popcorn seed (Field $\mathrm{C}$ hereafter referred to as SEEDTRT). Results from each dataset are reported after a demonstration of spatial correlation of relative elevation (RE) with other variables.

\section{Field A: Topographical terrain attributes}

Terrain attributes in Field A were suspected to be correlated with yield. Correlation between yield and terrain were demonstrated by measurements taken from a 160-ha field with highest level of elevation data quality available. Data included 286 survey-quality and 1,068 RTK-GNSS survey measurements combined into a single file (Figure 1). The survey-quality data were electronically collected including distance from observer and angles between base station and each marked location such that elevation could be calculated. Eight locations were measured by both survey and RTK-GNSS to align measurements, resulting in 1,346 elevation observations.

A total of 3,859 electrical conductivity (EC) measurements were georeferenced on 20-m transects. Topography, EC and yield data were recombined into a single dataset resulting in 1,075 observations. The final number of observations were less than the most sparse data layer $(\mathrm{N}=1,346)$ because not all data layers had observations within reasonable vicinity (for discussion of disparate spatial data layer assimilation see Griffin et al. 2007).

\section{<FIGURE 1 about here>}

Although no deliberate on-farm experiment was available for this field, these data were useful to demonstrate spatial correlation among yield and elevation variables. Univariate and bivariate Moran's I tested global spatial autocorrelation between variables and spatially-weighted average of immediate neighboring observations as defined by spatial weights matrices. One of the first steps in exploratory spatial data analysis (ESDA) is evaluation of Moran's I tests for global spatial autocorrelation in a random variable (Anselin, 1988; Cliff and Ord, 1981): 


$$
I=\frac{n}{S_{o}} \frac{x^{\prime} W x}{x^{\prime} x}
$$

where $\mathbf{x}$ is an $n$ by 1 vector of a random variable as deviations from the mean, $\mathbf{W}$ is an $n$ by $n$ spatial weights matrix described earlier in relation to spatial process models and $S_{o}$ is the sum of the elements of W (Anselin, 1988; Cliff and Ord, 1981). Moran's I is a spatial correlation coefficient not strictly bounded between $[-1,1]$ but rather $\left[\frac{1}{\Lambda_{(n-1)}}, \frac{1}{\Lambda_{(1)}}\right]$ where $\Lambda_{(n-1)}$ and $\Lambda_{(1)}$ are the minimum and maximum eigenvalues of $\mathbf{W}$, respectively. Moran's I can comfortably be interpreted as a correlation coefficient (Cliff and Ord, 1981; Anselin, 1988). Positive values of Moran's I are interpreted as high (low) values having neighbors of high (low) values, whereas negative values signify that high and low value observations occur as neighbors. Near-zero, i.e. not statistically significantly different from zero, Moran's I value signifies a random spatial distribution. Rather than considering a random variable with a spatially-weighted average of the same random variable, the bivariate Moran's I considers a random variable $\left(\mathrm{x}_{\mathrm{k}}\right)$ with the spatially-weighted average, or lag, of another random variable ( $\left.\mathrm{x}_{1}\right)$ (Eq. 9).

$$
I=\frac{n}{S_{o}} \frac{x_{k}^{\prime} W x_{l}}{x_{k}^{\prime} x_{k}}
$$

Although yield (YIELD), elevation (ELEV) and electrical conductivity (EC) had expected high levels of spatial autocorrelation ( $\mathrm{I}=0.70,0.97$ and 0.81 , respectively), relative elevation (RE) has relatively small Moran's I (0.07) but statistically different from zero (Table 1). Moran's I estimation is sensitive to the connectedness of the spatial interaction structure, $\mathbf{W}_{\mathbf{1}}$, (Bell and Bockstael 2000) used to calculate the cross-regressive term $\boldsymbol{W}_{1} \boldsymbol{E}$. The relatively limited geographic proximity that observations were considered neighbors caused micro-scale changes in RE to influence spatial autocorrection metrics. If greater connectedness, i.e. larger proximity, were used to define the spatial interaction structure, then higher Moran's I values would have been expected for RE. The bivariate Moran's I values between YIELD and ELEV $(\mathrm{I}=0.21)$ and EC (I=-0.35) showed moderate spatial association. Spatial autocorrelation between 
$\mathrm{RE}$ and the other variables were relatively small, but statistically significantly different from zero. Note that Table 1 is not necessarily required to be symmetric.

Table 1. Univariate and bivariate Moran's I test statistic for select random variables

\begin{tabular}{lllll} 
& \multicolumn{4}{c}{ Random Variable } \\
\cline { 2 - 5 } Spatially lagged variable & YIELD & ELEV & EC & RE \\
\hline YIELD & 0.70 & 0.21 & -0.35 & 0.05 \\
ELEV & 0.21 & 0.97 & -0.54 & 0.10 \\
EC & -0.35 & -0.54 & 0.81 & -0.08 \\
RE & 0.03 & 0.07 & -0.05 & 0.07 \\
\hline
\end{tabular}

$\mathrm{N}=1,075$

Null of no spatial autocorrection rejected at 5\% level for all 16 Moran's I tests

Although bivariate Moran's I for RE with other values were significantly different from zero, magnitudes of spatial autocorrelation were relatively small. Low levels of spatial autocorrelation indicated RE may be a candidate explanatory variable in aspatial models. When explanatory variables were spatially autocorrelated with the dependent variable, itself or other explanatory variables, residuals from aspatial regression models will be spatially autocorrelated resulting in inefficient aspatial estimation.

\section{Field B: Popcorn seed treatments, SEEDTRT}

Seven combinations of seed-applied insecticides and fungicides were compared on irrigated popcorn production in pseudo-replicated strip-trial experimental design for SEEDTRT in Tazewell County, Illinois, USA (Griffin et al. 2008). The 10-ha experiment (Figure 2) was planted with two passes of an 8-row planter with the control treatment (CHECK) between each of the six treatments and both sides of the experiment. Each treatment strip was harvested by two combine harvester passes.

<FIGURE 2 about here>

Treatment $\mathrm{X} 1$ and $\mathrm{X} 2$ were two recommended rates of the same insecticide. Treatment $\mathrm{X} 3$ was the fungicide. Treatments $\mathrm{X} 4$ and $\mathrm{X} 5$ were combinations of $\mathrm{X} 3$ with $\mathrm{X} 1$ and $\mathrm{X} 2$, respectively. Treatments $\mathrm{X} 6$ and $\mathrm{X} 7$ were two recommended rates of a second insecticide. The 
farmer's prior expectations included Treatment $\mathrm{X} 7$ dominating other treatments from a priori experiences. Therefore, Treatment $\mathrm{X} 7$ was the reference that other treatments were compared.

Full model specification (FULL) included binary variables for treatments (Xi), soil binary variables $(\mathrm{Si})$, elevation $(\mathrm{E})$, elevation squared $(\mathrm{E} 2), \mathrm{RE}$ and interaction terms between elevation and treatments (EXi). Second model specifications (EL) omitted RE. The WE model specification was the same as FULL except RE variable was replaced by cross-regressive variable, WE. Fourth model specification (RE) included RE but omitted all other topography variables.

In FULL and WE model specifications, aspatial results indicated Treatment X6 was statistically different from the control treatment, while aspatial estimation of RE model indicated Treatments X1, X3, X4 and X6 were statistically different from the control (Table 2). Results from SEM estimation were similar for models given that Treatments X2, X3, X4 and X6 statistically different from Treatment X7 for FULL, EL and WE models. Treatments X4 and X6 were statistically significant under the RE model specification.

Rankings within SEM models more closely resembled prior farmer expectations (Griffin et al., 2008) than OLS when evaluated at mean elevation (Table 3). The SEM model dominated OLS for FULL, EL and WE models. The RE model resulted in different agronomic rankings with Treatment X6 ranked second. The FULL and WE model specifications produced the same agronomic rankings for both OLS and SEM estimation. Although the RE model specification did not dominate the other models based on AIC, the inclusion of the RE variable in the FULL model was beneficial to the overall model fit for both OLS and SEM estimation based on AIC. Since elevation by treatment interaction terms was usually significant under SEM estimation, treatment rankings were sensitive to elevation and terrain position. 


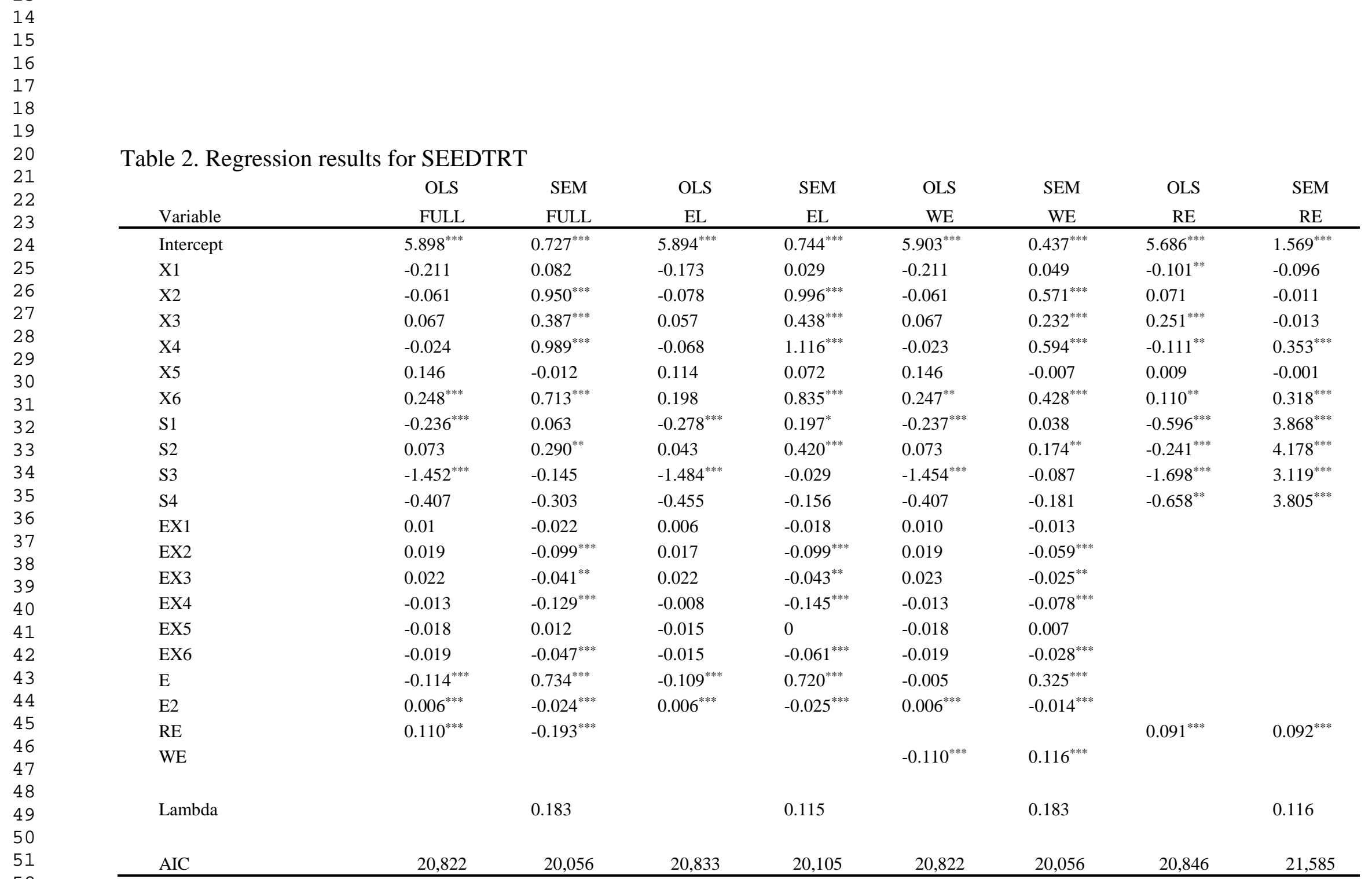

Significance denoted at $1,5,10 \%$ levels by $*, * *$ and $* * *$, respectively 


\begin{tabular}{|l|c|c|c|c|c|c|c|c|}
\hline & OLS & SEM & OLS & SEM & OLS & SEM & OLS & SEM \\
\hline FULL & FULL & EL & EL & WE & WE & RE & RE \\
\hline X1 & 4 & 5 & 4 & 5 & 4 & 5 & 5 & 3 \\
\hline X2 & 7 & 7 & 6 & 7 & 7 & 7 & 6 & 7 \\
\hline X3 & 1 & 2 & 3 & 2 & 3 & 2 & 3 & 5 \\
\hline X4 & 6 & 6 & 7 & 6 & 6 & 6 & 7 & 1 \\
\hline X5 & 5 & 3 & 5 & 4 & 5 & 3 & 4 & 4 \\
\hline X6 & 2 & 1 & 2 & 1 & 2 & 1 & 2 & 2 \\
\hline
\end{tabular}

\section{Field C: Soybean seeding rates, SOYSEED}

Five soybean seeding rates were replicated four times in a 19-ha strip-trial design two harvester passes wide per treatment in Montgomery County, Indiana, USA (Griffin et al. 2008). Seeding rates included very low rates $(197,600)$ to relatively high rates $(395,200)$ in increments of 49,400 seeds ha ${ }^{-1}$. Elevation data were collected via RTK-GNSS enabled automated guidance on the planter tractor (Figure 3). Yields were reported in $\mathrm{Mg} \mathrm{ha}^{-1}$.

\section{<FIGURE 3 about here>}

Full model specification (FULL) included seeding rate, rate squared, elevation, elevation squared, RE and interaction terms between rate and elevation (Table 4). For comparison, one model specification omitted all topography variables and included only seeding rate and its square (POP). The WE model specification was the same as FULL except RE variable was replaced by cross-regressive variable WE. The remaining model specification, EL, dropped RE from FULL.

Small changes in agronomically optimal seeding rates were observed between SEM and other model specifications. When agronomically-optimal seeding rates from any model specification were applied, estimated economic returns were similar to SEM with full model specification. For economic analyses, choice of estimator impacted the optimal population decision. In several model specifications, economic analyses using aspatial regression results calculated optimal seeding rate below the range of rates tested in the experiment. In these cases, the range of seeding rates was constrained to be within the vicinity of 197,600 to 395,200 seeds ha ${ }^{-1}$ range. 
Agronomically-optimal rates similar to current practices were estimated with OLS, but unconstrained economic analysis did not result in feasible solutions.

The AIC goodness-of-fit rankings for SEM resulted in FULL and WE model specifications being superior followed by EL model. With OLS, the AIC rankings held FULL superior to WE and WE superior to EL. The AIC value for RE and POP model specifications were identical, indicating that RE variable on its own was not beneficial to the model in this case. The SEM model dominated the aspatial and cross-regressive models in every model specification. Overall, regression model diagnostics indicated that RE models were not useful in this dataset. 
Table 4. SOYSEED regression results

\begin{tabular}{|c|c|c|c|c|c|c|c|c|c|c|}
\hline & OLS & SEM & OLS & SEM & OLS & SEM & OLS & SEM & OLS & SEM \\
\hline Variable & FULL & FULL & EL & EL & WE & WE & $\mathrm{RE}$ & $\mathrm{RE}$ & & \\
\hline Constant & $3.686^{* * * *}$ & 0.022 & $3.676^{* * *}$ & 0.015 & $3.603^{\text {*** }}$ & 0.022 & 4.136 & $0.120^{* *}$ & 4.134 & $0.124^{* *}$ \\
\hline POP & 0.001 & $0.059^{* * *}$ & 0.000 & $0.058^{* * *}$ & $-0.052^{* *}$ & $0.059^{* * * *}$ & 0.003 & $0.072^{* * *}$ & 0.003 & $0.072^{* * * *}$ \\
\hline POP_SQ & $0.000^{*}$ & $0.000^{* * * *}$ & $0.000^{*}$ & $0.000^{* * *}$ & 0.000 & $0.000^{* * * *}$ & 0.000 & $0.000^{* * * *}$ & 0.000 & $0.000^{* * * *}$ \\
\hline NELEV & $0.116^{* * * *}$ & $0.132^{* * *}$ & $0.117^{* * *}$ & $0.133^{* * *}$ & $0.184^{* * *}$ & $0.181^{* * * *}$ & & & & \\
\hline E2 & $-0.005^{* * * *}$ & $-0.005^{* * * *}$ & $-0.005^{* * * *}$ & $-0.005^{* * *}$ & $-0.005^{* * * *}$ & $-0.005^{* * * *}$ & & & & \\
\hline POP_ELV & $0.000^{* * * *}$ & 0.000 & $0.000^{* * *}$ & 0.000 & $0.000^{* * *}$ & 0.000 & & & & \\
\hline $\mathrm{RE}$ & $0.051^{* * * *}$ & $0.048^{* * *}$ & & & & & -0.022 & $0.011^{* * *}$ & & \\
\hline WE & & & & & $-0.061^{\text {**** }}$ & $-0.048^{* * * *}$ & & & & \\
\hline Lambda & & 0.375 & & 0.375 & & 0.375 & & 0.347 & & 0.347 \\
\hline AIC & 23,954 & 21,461 & 23,969 & 21,479 & 23,960 & 21,461 & 24,992 & 21,731 & $\begin{array}{l}24,99 \\
2\end{array}$ & 21,731 \\
\hline
\end{tabular}

Significance denoted at $1,5,10 \%$ levels by $* * *$ and $* * *$, respectively 


\section{Multicollinearity condition number of Field B and Field C}

Compared to full elevation models that include elevation, its square and interaction terms, model specifications with RE as the only topographic variable had minimal multicollinearity condition numbers. Condition numbers are based on the ratio of largest and smallest eigenvalues, $\Lambda$, of the matrix (Eq. 10) (Greene, 2012). Condition numbers larger than 20 were considered large meaning that the matrix is nearly singular (Greene, 2012). However, multicollinearity typically is not a problem if coefficients remain robust. The larger the condition number, the more computationally difficult it is to invert the matrix. In regressions, the level of multicollinearity in matrices of explanatory variables $\mathbf{X}$ was of interest, so the condition number $(\mathrm{CN})$ of the cross product of $\mathbf{X}\left(\boldsymbol{X}^{\prime} \boldsymbol{X}\right)$ was calculated.

$C N=\left[\frac{\Lambda_{\max }}{\Lambda_{\min }}\right]^{0.5}$

where $\mathrm{CN}$ is condition number and $\Lambda$ are eigenvalues of $\mathbf{X}$.

Full model specification (FULL) including all topography variables (RE, elevation, elevation squared) and EL models had the same multicollinearity number (Table 5). For both SEEDTRT and SOYSEED trials, dropping the RE variable leaving only the seeding rate and its square as only explanatory variables in the model, no difference in condition number was detected. The classic cross regressive term, WE, increased multicollinearity condition numbers for both data sets. Model specifications including only the relative elevation variable, RE, substantially reduced the multicollinearity condition number compared to model specifications using elevation, its square and interaction terms (EL), i.e. the FULL model (Table 5).

Table 5. Multicollinearity Condition Number for selected studies and model specifications

\begin{tabular}{lcc} 
Model & SEEDTRT & SOYSEED \\
\hline FULL & 45 & 133 \\
EL & 45 & 133 \\
WE & 82 & 154 \\
RE & 7 & 92 \\
No topo & 7 & 92 \\
\hline
\end{tabular}




\section{Conclusions}

Cross-regressive variables were useful in modeling field-scale precision agriculture datasets; however, they did not globally dominate status quo models that explicitly account for spatial effects. Rather than substituting for conventional terrain variables, WE and RE complemented those variables in field examples evaluated. Proposed relative elevation (RE) variables did not strictly dominate nor were strictly dominated by other model specifications including conventional topography variables. This was demonstrated by the AIC regression diagnostics and low bivariate Moran's I value for RE relative to other continuous variables. Both hypotheses were supported. Thus, the conclusions of this research are:

1) Cross regression relative elevation variables facilitated testing of treatment differences because they did not aggravate multicollinearity the way that elevation and its derivatives often do.

2) The next step is to try cross regression with a wider range of data. For example, ongoing research is evaluating cross-regressive variables explicitly for modeling treatment edge effects in field-scale on-farm research.

3) Cross regression should be considered for incorporation into decision tools that use yield monitoring data.

\section{References}

Anselin, L. (1988). Spatial Econometrics: Methods and Models, Dordrecht, Netherlands: Kluwer Academic Publishers.

Anselin, L. (2001). Spatial Effects in Econometric Practice in Environmental and Resource Economics. American Journal of Agricultural Economics 83(3), 705-710.

Anselin, L. (2002). Under the hood Issues in the specification and interpretation of spatial regression models. Agricultural Economics. 27(3), 247-267.

Anselin, L., Bongiovanni, R., \& Lowenberg-DeBoer, J. (2004). “A Spatial Econometric Approach to the Economics of Site-Specific Nitrogen Management in Corn Production," American Journal of Agricultural Economics, 86(3), 675-687.

Arbia, G. (2014). A Primer for Spatial Econometrics with Applications in R. New York, NY, USA: Palgrave MacMillan. 
Bell, K. P. \& Bockstael, N. E. (2000). Applying the Generalized-Moment Estimation Approach to Spatial Problems Involving Micro-level Data. The Review of Economics and Statistics, 82(1), 72-82.

Bishop,T. F. A. \& McBratney, A. B. (2002). Creating Field Extent Digital Elevation Models for Precision Agriculture. Precision Agriculture, 3(1), 37-46.

Clark, R. L. \& Lee, R. (1998). Development of Topographic Maps for Precision Farming with Kinematic GPS. Transactions of the ASAE, 41(4), 909-916.

Cliff, A.D. \& Ord, J.K. (1981) Spatial processes: Models and applications. London, UK: Pion Limited.

Coble, K., Ferrell, S.L., Mishra, A., \& Griffin, T.W. (2018). Big Data in Agriculture: A Challenge for the Future. Applied Economics Perspectives and Policy, 40(1), 79-96.

Dubin, R.A. (2003). Robustness of Spatial Autocorrelation Specifications: Some Monte Carlo Evidence. Journal of Regional Science, May 2003, p. 221-248.

Florax, R. \& Folmer, H. (1992). Specification and Estimation of Spatial Linear Regression Models: Monte Carlo Evaluation of Pre-Test Estimators. Regional Science and Urban Economics, 22, 405-432.

Florax, R.J.G.M., Voortman, R.L., and Brouwer, J. (2002). Spatial Dimensions of Precision Agriculture: A Spatial Econometric Analysis of Millet Yield on Sahelian Coversands. Agricultural Economics. 27(3), 425-443.

Garrido, M. S., de Lacy, M. C., Ramos, M.I., Borque, M. J., \& Susi, M. 2019. Assessing the accuracy of NRTK altimetric positioning for precision agriculture: test results in an olive grove environment in Southeast Spain. Precision Agriculture, 20(3), 461-476.

Greene, W. H. (2012). Econometric Analysis 7th Ed., Pearson Education, Upper Saddle River, New Jersey, USA: Prentice Hall. 1,232 pp.

Griffin, T. W., Brown, J. P., \& Lowenberg-DeBoer, J. (2007). Yield Monitor Data Analysis Protocol: A Primer in the Management and Analysis of Precision Agriculture Data. Purdue University. Available at https://ssrn.com/abstract=2891888 Accessed 16 November 2019

Griffin, T. W., Dobbins, C. L., Vyn, T. J, Florax, R. J. G. M., \& Lowenberg-DeBoer, J. (2008). Spatial analysis of yield monitor data: case studies of on-farm trials and farm management decision making. Precision Agriculture, 9(5), 269-283.

Griffin, T.W., Mark, T.B., Dobbins, C.L., \& Lowenberg-DeBoer, J. (2014). Estimating Whole Farm Costs of Conducting On-farm Research: A Linear Programming Approach. International Journal of Agricultural Management. 4(1), 21-27. 
Griffin, T.W. (2010). The Spatial Analysis of Yield Data. In M. Oliver (Ed.) Geostatistical Applications for Precision Agriculture. Dordrecht, Netherlands: Springer. 295p.

Griffin, T.W., \& Yeager, E.A. (2019). How quickly do farmers adopt technology? A duration analysis. In J.V. Stafford (Ed.) Precision agriculture '19. $12^{\text {th }}$ European Conference on Precision Agriculture. Wageningen, The Netherlands: Wageningen Academic Publishers, pp 843-849.

Hartsock, N. J., Mueller, T. G., Karathanasis, A. D., \& Cornelius, P. L. (2005). Interpreting Soil Electrical Conductivity and Terrain Attribute Variability with Soil Surveys. Precision Agriculture, 6(1), 53-72.

Hurley, T. M., Oishi, K., \& Malzer, G. L. (2005). Estimating the potential value of variable rate nitrogen applications: A comparison of spatial econometric and geostatistical models. Journal of Agricultural and Resource Economics, 30(2), 231-249.

Jiang, P. \& Thelen, K.D. (2004). Effect of Soil and Topographic Properties on Crop Yield in a North-Central Corn-Soybean Cropping System. Agronomy Journal, 96(1), 252-258.

Kaspar, T.C., Pulido, D. J., Fenton, T. E., Colvin, T. S., Karlen, D. L., Jaynes, D. B., et al. (2004). Relationship of Corn and Soybean Yield to Soil and Terrain Properties. Agronomy Journal, 96(3), 700-709.

Kelejian, H. H. \& Prucha, I. R. (1999). A Generalized Moments Estimator for the Autoregressive Parameter in a Spatial Model. International Economic Review, 40, 509-533.

Kelejian, H. H., \& Prucha, I. R. (2010). Specification and Estimation of Spatial Autoregressive Models with Autoregressive and Heteroskedastic Disturbances. Journal of Econometrics, 157(1), 53-67.

Kelejian, H. \& Prucha, I. (1998). A Generalized Spatial Two Stage Least Squares Procedure for Estimating a Spatial Autoregressive Model with Autoregressive Disturbances. Journal of Real Estate Finance and Economics, 17(1), 99-121.

Kravchenko, A.N., Bullock, D.G., \& Boast, C.W. (2000). Joint Multifractal Analysis of Crop Yield and Terrain Slope. Agronomy Journal, 92(6), 1279-1290.

Lambert, D.M., Lowenberg-DeBoer, J., \& Bongiovanni, R. (2004). A Comparison of Four Spatial Regression Models for Yield Monitor Data: A Case Study from Argentina. Precision Agriculture, 5:579-600.

LeSage, J. \& Pace, R.K. (2009) Introduction to Spatial Econometrics 1st Edition. Boca Raton, FL, USA: Taylor \& Francis. 394 pp.

Liu, Z., Griffin, T.W., Kirkpatrick, T.L., \& Monfort, W.S. (2015). Spatial Econometric Approaches to Site-Specific Nematode Management Strategies. Precision Agriculture. 16(5), 587-600. 
Long, D.S. \& McCallum, J.D. (2015). On-combine, multi-sensor data collection for post-harvest assessment of environmental stress in wheat. Precision Agriculture, 16(5), 492-504.

Miao, Y., Mulla, D.J., \& Robert, P.C. (2006). Spatial Variability of Soil Properties, Corn Quality and Yield in Two Illinois, USA Fields: Implications for Precision Corn Management. Precision Agriculture, 7(1), 5-20.

Miller, N.J., Griffin, T.W., Ciampitti, I., \& Sharda, A. (2019). Farm Adoption of Embodied Knowledge and Information Intensive Precision Agriculture Technology Bundles. Precision Agriculture, 20(2), 348-361.

Papadakis, J. S. (1937). Methode statistique pour des experiences sur champs ('Statistical Methods for Field Experiments.) Bulletin de 1'Institut de 1'Amelioration des Plantes, Thessaloniki (Greece), p. 23.

Selle, M.L., Steinsland, I., Hickey, J. M., \& Gorjanc, G. (2019). Modelling spatial variation in agricultural field trials with INLA. bioRxiv. doi:10.1101/612036

Sudduth, K.A., Drummond, S.T., \& Myers, D.B. (2012). Yield Editor 2.0: Software for automated removal of yield map errors. Paper no. 121338343. St. Joseph, MI, USA: ASABE. Retrieved from http://extension.missouri.edu/sare/documents/ASABEYieldEditor2012.pdf Accessed 16 November 2019.

Thomas, I.A., Jordan, P., Shine, O., Fenton, O., Mellander, P.-E., Dunlop, P., et al. (2017). Defining optimal DEM resolutions and point densities for modelling hydrologically sensitive areas in agricultural catchments dominated by microtopography, International Journal of Applied Earth Observation and Geoinformation, 54: 38-52.

Trevisan, R. G., Bullock, D.S., \& N.F. Martin. (2019). Site-Specific Treatment Responses in OnFarm Precision Experimentation. Preprints, 10.20944/preprints201902.0007.v1. Accessed 18 November 2019. 


\section{Legend} elevation ( $m$ )

- $\quad 210.1-211.6$

- $211.7-212.7$

- $212.8-213.8$

- $\quad 213.9-215.1$

- $215.2-216.6$

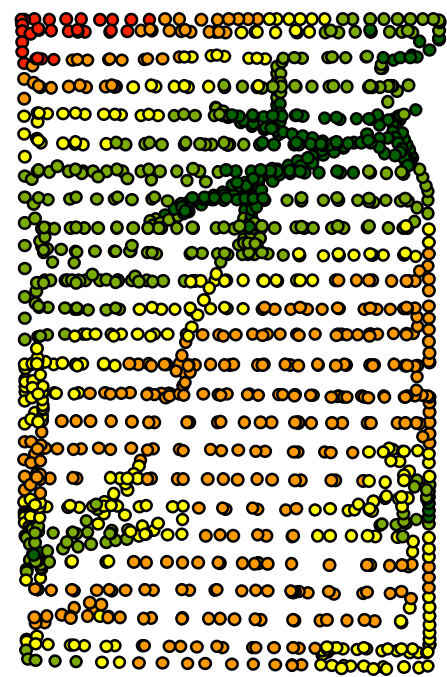

Figure 1. Field A elevation 


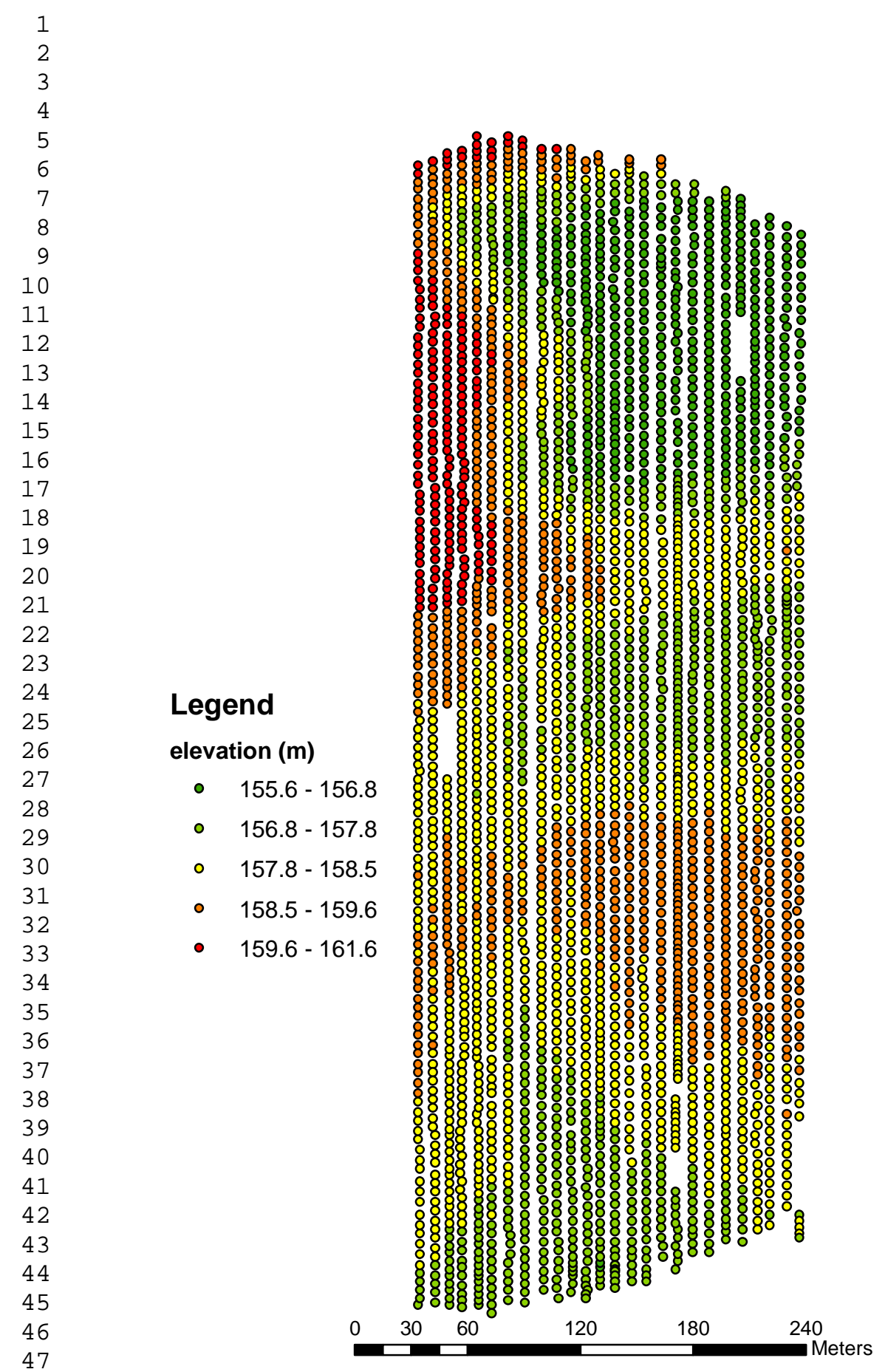

48

49

50

51

52

53

54

55

56

57

58

59

60

61

62

63 


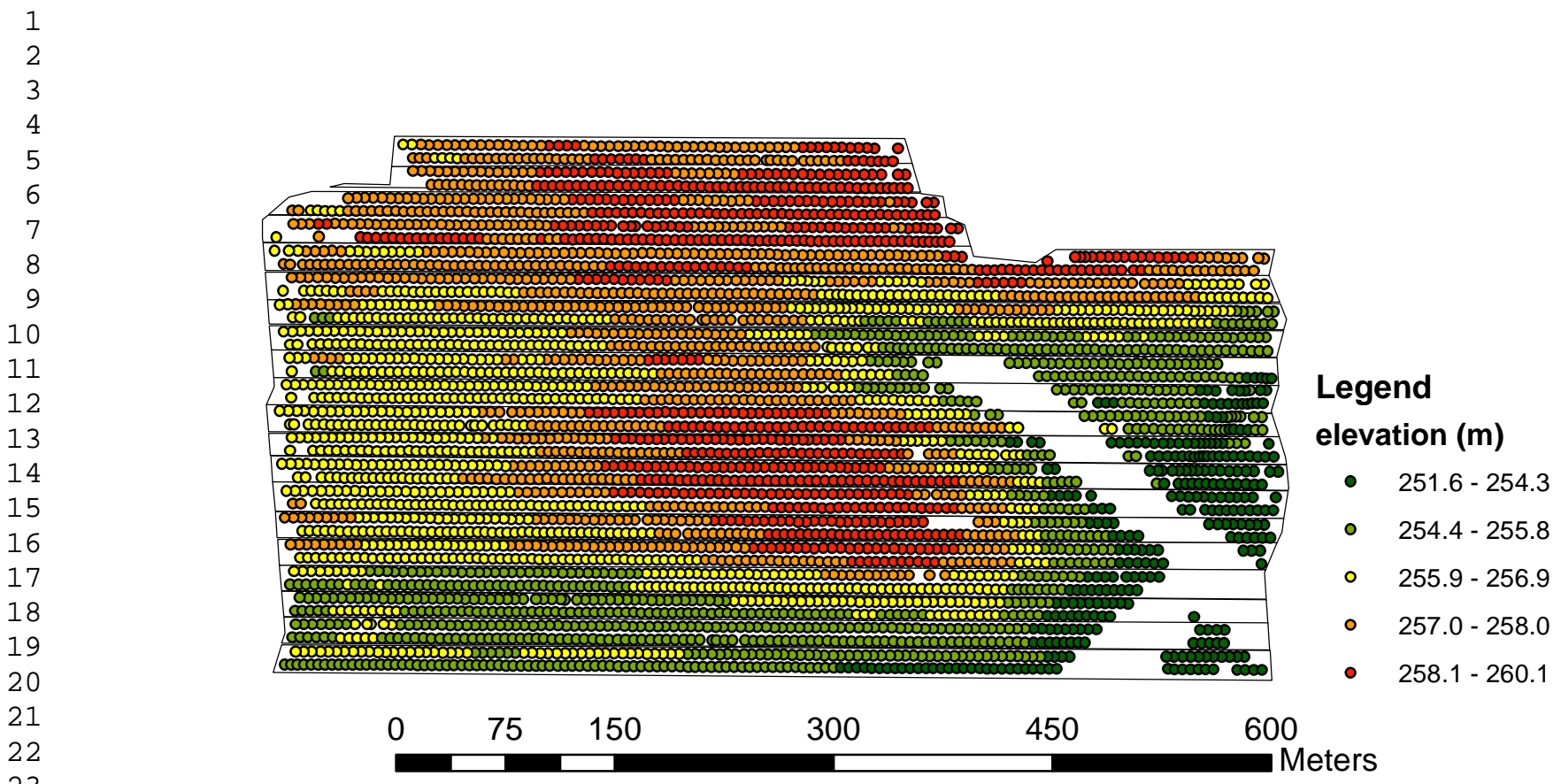

Figure 3. Field C elevation 